\title{
A Fast Algorithm for Treating Dielectric Discontinuities in Charged Spherical Colloids
}

\author{
Zhenli XU* \\ (Department of Mathematics, and Institute of Natural Sciences, Shanghai Jiao Tong University, Shanghai 200240, China)
}

Received 22 June 2011 / Revised 28 July 2011 / Accepted 29 July 2012

\begin{abstract}
Electrostatic interactions between multiple colloids in ionic fluids are attracting much attention in studies of biological and soft matter systems. The evaluation of the polarization surface charges due to the spherical dielectric discontinuities poses a challenging problem to highly efficient computer simulations. In this paper, we propose a new method for fast calculating the electric field of spaced spheres using the multiple reflection expansion. The method uses a technique of recursive reflections among the spherical interfaces based on a formula of the multiple image representation, resulting in a simple, accurate and close-form expression of the surface polarization charges. Numerical calculations of the electric potential energies of charged spheres demonstrate the method is highly accurate with small number of reflections, and thus attractive for the use in practical simulations of related problems such as colloid suspension and macromolecular interactions.
\end{abstract}

Key words: electrostatic polarization, method of images, Green's functions, colloidal suspensions, dielectric spheres.

\section{Introduction}

The understanding of the solute and solvent polarizability is crucial to theoretical and computational studies of electrostatic interactions of charged systems in many applications of soft matter physics, biophysics, and colloidal science. It has been shown the polarization potential has an important effect on the distribution of ions surrounding a colloidal sphere, which significantly influences the overcharging of the Thomson sphere (Messina, 2009). Recent simulations also demonstrate the surface polarization (Rescics and Linse, 2008) results in an enhanced attractive potential for spherical macroions at long separation, and thus it could be an important component for the longranged attractions of like-charge objects, for which the mechanism of attractions remains a debating problem (Boroudjerdi et al., 2005; French et al., 2010; Levin, 2002; Messina, 2009). The mean-field PoissonBoltzmann theory fails to explain the phenomena of the overcharging and the like-charge attraction as it does not account for the strong ion correlations between multivalent counterions. Computer simulations such as Monte Carlo (Frenkel and Smit, 2002) become important tools to understand these mysterious phenomena, motivating a requirement of analytical or fast numer-

\footnotetext{
${ }^{*}$ Corresponding author.

E-mail: xuzl@sjtu.edu.cn
}

ical solutions of electrostatic polarization problems in various dielectric environments.

A number of theoretical studies (Bhuiyan et al., 2009; Boda et al., 2004; Linse, 2008; Messina, 2002; Torrie et al., 1982; Tyagi et al., 2010; Wang and Ma, 2009) have been conducted to investigate the polarization effect of dielectric discontinuities to the counterion distributions in the electric double layer. A precise representation of the polarization potential limits on simple geometries such as a single planar or spherical interface. For one dielectric sphere embedded in a uniform background of dissimilar dielectric, the multipole series expansion in terms of spherical harmonics is often used (Messina, 2002). The obtained Kirkwood series (Kirkwood, 1934) is known to behave a slow convergence when the source is close to the boundary, and thus alternative ways such as the methods of image charges have been widely considered (Abagyan and Totrov, 1994; Cai et al., 2007; Friedman, 1975; Linse, 1986). The presence of multiple inclusions poses a difficulty for the treatment of the electrostatics, even for the interaction of two spheres, though its solution is significant for the study of colloid-colloid interactions and protein-protein interactions in biological systems. Phillies (Phillies, 1974) developed an analytical solution for estimating electrostatic interaction energy of two globular proteins of arbitrary charge distributions, which was improved in McClurg and Zukoski (1998) 
by using explicit re-expansion operators. Allen and Hansen (2002) proposed a density functional method to study the solution of two charges in either two spherical dielectrics. Recently, Linse (2008) studied the electrostatics of any number of multipoles each in a spherical dielectric, in particular, simplified expressions of two dielectric cavities have been provided, and used to investigated the potential of mean force between two likecharged macroions (Rescics and Linse, 2008). Lotan and Head-Gordon (2006), and Yap and Head-Gordon (2010) developed analytical multipole methods to iteratively compute the surface charges of multiple proteins in the presence of ionic solvent with a Debye-Hückel treatment. Analytical and numerical investigations of the nonlinear Poisson-Boltzmann equations (Neu, 1999; Qiao et al., 2006; Xing, 2011) are also performed for wall-mediated electrostatic forces between multiple objects. It should be stated that the generalized Born method can also be used to calculate the dielectric effects with a simple analytical expression. Its extension in the colloidal science could be very interesting, although the accuracy could become an issue and the parameters involved in the expression should be calibrated before the use due to the phenomenological nature.

Unlike the multipole expansion with spherical harmonics, in this paper, we propose a method of images for the treatment of the electrostatics in the presence of dielectric multispheres. In the approximation with image charges to the case of several objects, the multiple reflections (MR) are often introduced, which recursively construct images by alternating the interfaces until convergence (Bhattacharya and Blawzdziewicz, 2002; Cheng and Greengard, 1998; Xu et al., 2011; Yang et al., 2002). The extension to multiple dielectric spheres is, however, not straightforward, because the polarization effect can not be simply approximated by an image charge. Approximate solutions based on integral equations for the line image charges were discussed by Lindell et al. (1993). The recently developed multiple-image method (Cai et al., 2007) for the line integral expression of the spherical polarization provides a high order of accuracy with several point images, based on which we use the MR to construct a multipole series in terms of image charges of multi-levels, and approximate the multi-spherical polarization at desired accuracy by varying the number of images and the number of reflections. The obtained expression is simple and efficient to compute, thus will be useful for molecular dynamics and Monte Carlo simulations of charged particles when the dielectric continuities play a reasonable role, such as colloid-colloid interactions and polyvalent ion-ion correlations. Potential applications of our results to other problems could be found in aforementioned review papers.

The present work focuses on the theoretical derivation of the image method and its accuracy test. The method can be naturally speeded up by using the fast multipole algorithm (Greengard and Rokhlin, 1987; Lu et al., 2006) as the image approximation transforms the inhomogeneous system into a system of Coulomb interactions in free space. Incorporating the proposed method together with the fast accelerating algorithm to Monte Carlo simulations, and thus their application in studying colloid-colloid interactions in electrolyte solutions with multivalent ions, will be the objective of the subsequent publications.

The organization of this paper is as follows. In section 2 , the theory and method of images are presented, including the cases of both one and multiple dielectric spheres. In section 3, numerical results and discussion are given. In section 4, discussion and conclusion are made.

\section{Method}

In this section, we will present the image representation of electrostatic potential induced by a point charge $q$ at $\mathbf{r}_{s}$ located outside $S$ spaced spheres with centers at $\mathbf{r}_{i}$, and radii $R_{i}$ for $i=1,2, \cdots, \mathrm{S}$. The source position is at the outside of the spheres. This representation gives the Green function of the system, containing the effect of the dielectric boundaries. The spherical interiors are characterized by dielectric permittivity $\varepsilon_{i}$, and the exterior by $\varepsilon_{\mathrm{ex}}$. For convenience the charges of the spheres are assumed to be zero, and if not, the superposition principle can be used to add its influence. Then the potential function $\Phi(\mathbf{r})$ is described by the Poisson equation,

$$
-\nabla \cdot \varepsilon(\mathbf{r}) \nabla \Phi(\mathbf{r})=4 \pi q \delta\left(\mathbf{r}-\mathbf{r}_{s}\right),
$$

where $\delta(\cdot)$ is the Dirac delta function, and $\varepsilon(\mathbf{r})$ is the piecewise constant dielectric function which takes $\varepsilon_{i}$ in the $i$ th sphere, and $\varepsilon_{\mathrm{ex}}$ in the background solvent. The solution of the Poisson equation can be written as the sum of the Coulomb potential and the surface polarization contribution, $\Phi(\mathbf{r})=q / \varepsilon_{\mathrm{ex}}\left|\mathbf{r}-\mathbf{r}_{\mathrm{s}}\right|+\Phi_{\mathrm{pol}}$, where $\Phi_{\text {pol }}$ is our main concern and will be discussed by the method of images below. We will first consider one sphere case, and then extend the results to treat multiple spheres.

The simplest case is the situation of only one sphere present, $S=1$. Suppose the spherical radius be $R$, and let us move the origin of the coordinates to the center of the sphere. We consider the electrostatic potential by using the spherical coordinates $\mathbf{r}=(r, \theta, \varphi)$, and the source position is $\mathbf{r}_{s}=\left(r_{s}, \theta_{s}, \varphi_{s}\right)$ for $r_{s}>R$. It is well known that the polarization potential can be described in terms of spherical harmonics (see for example Refs. (Cai et al., 2007; Messina, 2002) for the derivation),

$$
\Phi_{\mathrm{pol}}(\mathbf{r})=\frac{q}{\varepsilon_{\mathrm{ex}}} \sum_{n=0}^{\infty} \frac{R^{2 n+1}}{\left(r r_{s}\right)^{n+1}} g(n) P_{n}(\cos \eta),
$$


where $\eta$ is the angle between the field point $\mathbf{r}$ and the source point $\mathbf{r}_{s}$, and $P_{n}(\cdot)$ is the Legendre polynomial of order $n$, and

$$
g(n)=-\gamma+\frac{\gamma}{\left(\varepsilon_{1} / \varepsilon_{\mathrm{ex}}+1\right) n+1},
$$

with $\gamma=\left(\varepsilon_{1}-\varepsilon_{\text {ex }}\right) /\left(\varepsilon_{1}+\varepsilon_{\text {ex }}\right)$. The series for the polarization is known as the Kirkwood series. A straightforward way to compute it is to truncate the infinite series into a finite sum. The convergence is, however, slow for charges near the spherical surface.

The method of images is a standard strategy for electrostatic problems, which is to find a simple representation (or approximation) of the Green's function in inhomogeneous media (Jackson, 2001). In the simplest case of the presence of only one planar interface which separates the space into two different media, the Green's function of the Poisson equation of this system can be exactly described as a sum of the potentials due to a unit point charge at the source position and a virtual charge at the mirror symmetric point. This virtual charge is naturally called the image charge, and its strength is determined by the dielectric constants of the two media. In regard to the spherical interface, the Kelvin image methods (Abagyan and Totrov, 1994; Friedman, 1975) have been widely used in early publications, see (Deng et al., 2007) for a comparable study of these methods, and the surveys (Hatlo and Lue, 2008; Lindell, 1993; Xu and Cai, 2011) for the role in various applications. The basic idea of the Kelvin image is to asymptotically approximate the coefficients of the Kirkwood series expansion, such that the resulted series happens to be the spherical harmonics expansion of the reciprocal distance between the Kelvin point and the source point. The obtained image representation for the Green's function is exact at the conductor limit of the bulk solvent, however, it is an approximation for a finite dielectric constant and considered inaccurate in many applications.

The alternative expression is through the line image representation (Cai et al., 2007; Lindell, 1992; Norris, 1995) of the reaction potential. Let us recall the multipole expansion of the reciprocal distance of any two points $\mathbf{r}_{1}$ and $\mathbf{r}_{2}$, given by,

$$
\frac{1}{\left|\mathbf{r}_{1}-\mathbf{r}_{2}\right|}=\sum_{n=0}^{\infty} \frac{r_{<}^{n}}{r_{>}^{n+1}} P_{n}\left(\cos \eta_{12}\right)
$$

where $\eta_{12}$ is the angle between the two vectors, and $r_{<}\left(r_{>}\right)$is the smaller (larger) one of $r_{1}$ and $r_{2}$. If we write $g(n)$ in terms of the Mellin transform,

$$
g(n)=\{M f\}(n)=\int_{0}^{\infty} t^{n-1} f(t) d t
$$

with $f(t)$ being expressed by the inverse Mellin transform,

$$
\begin{aligned}
f(t) & =\left\{M^{-1} g\right\}(t) \\
& =-\gamma \delta(t-1)+\frac{\gamma t^{\sigma}}{\varepsilon_{1} / \varepsilon_{\mathrm{ex}}+1} H(1-t) .
\end{aligned}
$$

Here, $\sigma=\varepsilon_{\text {ex }} /\left(\varepsilon_{1}+\varepsilon_{\text {ex }}\right)$ and $H(\cdot)$ is the Heaviside step function. Let us define the Kelvin image inverse point by $\mathbf{r}_{K}=\mathbf{r}_{s} R^{2} / r_{s}^{2}$, and so that $r_{K}=R^{2} / r_{s}$. Now if we substitute the Mellin transform of $g(n)$ in the expression (2), we can write the polarization in the form of Eq. (4),

$$
\Phi_{\mathrm{pol}}(\mathbf{r})=\frac{q}{\varepsilon_{\mathrm{ex}}} \int_{0}^{\infty} d t \frac{R f(t)}{r_{s} t} \sum_{n=0}^{\infty} \frac{\left(r_{K} t\right)^{n}}{r^{n+1}} P_{n}(\cos \eta) .
$$

Considering $f(t)=0$ when $t>1$ and $r_{K} t<r$ when $t \leq 1$, we see the summation involved in the above expression is in the form of Eq. (4), and thus we rewrite the polarization potential into the sum of a point image and a line image by substituting the expression of the strength function $f(t)$,

$$
\Phi_{\text {pol }}(\mathbf{r})=\frac{q_{K}}{\varepsilon_{\text {ex }}\left|\mathbf{r}-\mathbf{r}_{K}\right|}+\int_{0}^{r_{K}} \frac{q_{\text {line }}(x)}{\varepsilon_{\text {ex }}|\mathbf{r}-\mathbf{x}|} d x,
$$

where $\mathbf{x}=x \mathbf{r}_{s} / r_{s}$, and the point and line images are respectively the contributions of the delta and Heaviside functions in Eq. (6) and their strengths are given by,

$$
q_{K}=-\frac{\gamma R}{r_{s}} q, \quad \text { and } \quad q_{\text {line }}(x)=\frac{\gamma \sigma q}{R}\left(\frac{r_{K}}{x}\right)^{1-\sigma} .
$$

Though Eqs. (2) and (8) are equivalent, the latter one provides a simpler way to find an efficient approximation once numerical integration is used to obtain a form of discrete point images (Cai et al., 2007; Lin et al., 2009). The $M$-point Gauss-Legendre quadrature is used to approximate the line integral, leading us to a total of $M+1$ images,

$$
\Phi_{\mathrm{pol}}(\mathbf{r})=\sum_{m=0}^{M} \frac{q_{m}}{\varepsilon_{\mathrm{ex}}\left|\mathbf{r}-\mathbf{x}_{m}\right|},
$$

where $q_{0}=q_{K}$ and $\mathbf{x}_{0}=\mathbf{r}_{K}$ are the Kelvin image's strength and position,

$$
q_{m}=\frac{\omega_{m}}{2} \frac{\gamma R q}{r_{s}}, \text { and } x_{m}=r_{K}\left(\frac{1-s_{m}}{2}\right)^{1 / \sigma},
$$

for $m=1, \cdots, M$, are the charges and positions of the discrete point images due to the line integral contribution, and $\left\{\omega_{m}, s_{m}, m=1,2, \cdots, M\right\}$ are the $M$-point Gauss weights and locations on the interval $[-1,1]$. As the total strength of the line image is equal to the inverse of the Kelvin image, at least two images have to be used to remain the electric neutrality. 
We now study the case of two inclusions of spherical shape with a point charge $q$ placed at $\mathbf{r}_{s}$ in the background, as schematically shown in Fig. 1. The multipole solution of the polarization potential in terms of spherical harmonics can be found in a recent paper (Rescics and Linse, 2008), and will not be present. As the computational cost of this spherical harmonics solution is high, we will reformulate a new multipole expansion in the form of image charges.

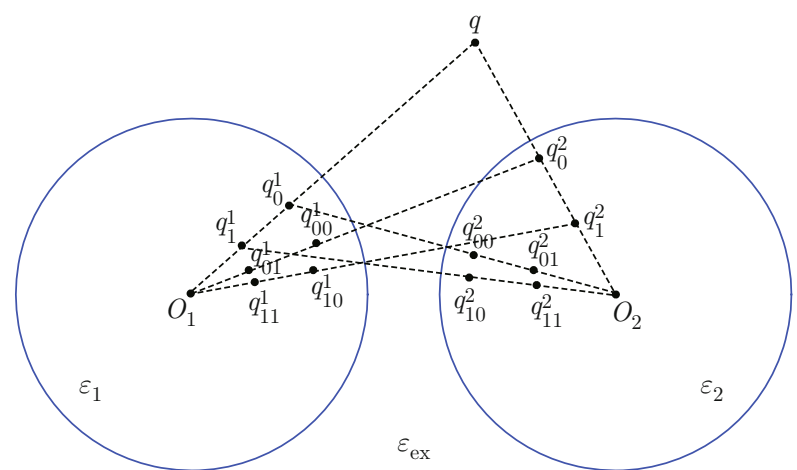

Fig. 1 An 2D illustration of image charges due to a point charge outside two separate spheres with $M=1$. The point charge induces an approximate dipole in each sphere. Each dipole produces an approximate quadrupole in alternating sphere. Higher order reflections are constructed iteratively in the same manner and not present.

Due to the dielectric boundaries, the charge will produce images within both spheres, which comprise the total potential as follows,

$$
\Phi^{(1)}=\sum_{m_{1}=0}^{M}\left(\frac{q_{m_{1}}^{1}}{\varepsilon_{\mathrm{ex}}\left|\mathbf{r}^{\prime}-\mathbf{x}_{m_{1}}^{1^{\prime}}\right|}+\frac{q_{m_{1}}^{2}}{\varepsilon_{\mathrm{ex}}\left|\mathbf{r}^{\prime \prime}-\mathbf{x}_{m_{1}}^{2^{\prime \prime}}\right|}\right)
$$

where the prime symbol represents the coordinates with the origin at $O_{1}$, while the double prime symbol is with the origin at $\mathrm{O}_{2}$, and the superscripts "1" and "2" represent the parameters of images within spheres 1 and 2 , respectively. The strengths and locations are given by the formula of a single sphere, for example, $q_{m_{1}}^{1}$ and $\mathbf{x}_{m_{1}}^{1^{\prime}}$ for $m_{1}=0, \cdots, M$ are determined by the images of the source $q$ due to the dielectric boundary of sphere 1, which are given by Eq. (10).

However, the presence of the image charges, $\left\{q_{m_{1}}^{1}\right\}$, does not consider the influence of the 2 nd sphere and thus the boundary conditions across this interface break down. Analogously, the images $\left\{q_{m_{1}}^{2}\right\}$ do not satisfy the boundary conditions on the 1 st spherical interface. Therefore, these images will create a polarization effect due to the dielectric discontinuity of the alternating sphere. Thus, the images' images will appear inside the sphere 2 with $\left\{q_{m_{1}}^{1}\right\}$ as source charges, and inside the sphere 1 with $\left\{q_{m_{1}}^{2}\right\}$ as source charges. This gives the potential of the second reflection,

$$
\begin{aligned}
\Phi^{(2)}= & \sum_{m_{1}=0}^{M} \sum_{m_{2}=0}^{M}\left(\frac{q_{m_{1} m_{2}}^{1}}{\varepsilon_{\mathrm{ex}}\left|\mathbf{r}^{\prime}-\mathbf{x}_{m_{1} m_{2}}^{1^{\prime}}\right|}+\right. \\
& \left.\frac{q_{m_{1} m_{2}}^{2}}{\varepsilon_{\mathrm{ex}}\left|\mathbf{r}^{\prime \prime}-\mathbf{x}_{m_{1} m_{2}}^{2^{\prime \prime}}\right|}\right),
\end{aligned}
$$

where $q_{m_{1} m_{2}}^{1}$ and $\mathbf{x}_{m_{1} m_{2}}^{1^{\prime}}$ for $m_{2}=0, \cdots, M$ are the image strengths and locations of the dielectric response within the 1 st sphere due to the charge $q_{m_{1}}^{2}$, and similarly, $q_{m_{1} m_{2}}^{2}$ and $\mathbf{x}_{m_{1} m_{2}}^{2^{\prime \prime}}$ for $m_{2}=0, \cdots, M$ are the images within the 2 nd sphere due to the charge $q_{m_{1}}^{1}$.

Again, these images' images will produce images in alternating spheres. Overall, the recursive reflections define the total polarization potential due to the source $q$ in the presence of the two spheres,

$$
\Phi_{\mathrm{pol}}(\mathbf{r})=\Phi^{(1)}+\Phi^{(2)}+\cdots,
$$

with the $p$ th order images being

$$
\begin{aligned}
\Phi^{(p)}= & \sum_{m_{1}=0}^{M} \cdots \sum_{m_{p}=0}^{M}\left(\frac{q_{m_{1} \cdots m_{p}}^{1}}{\varepsilon_{\mathrm{ex}}\left|\mathbf{r}^{\prime}-\mathbf{x}_{m_{1} \cdots m_{p}}^{1^{\prime}}\right|}+\right. \\
& \left.\frac{q_{m_{1} \cdots m_{p}}^{2}}{\varepsilon_{\mathrm{ex}}\left|\mathbf{r}^{\prime \prime}-\mathbf{x}_{m_{1} \cdots m_{p}}^{2^{\prime \prime}}\right|}\right),
\end{aligned}
$$

where the parameters of images are determined by the $(p-1)$-th order images recursively. We thus construct an exact series representation for the electric potential, which can be approximated by truncating it at a finite term, $\Phi_{\text {pol }}(\mathbf{r})=\Phi^{(1)}+\Phi^{(2)}+\cdots+\Phi^{(P)}$. The accuracy of the approximation depends on the number of the used terms, $P$, and the distance between two spheres.

In Fig. 1, we schematically illustrate the images up to the second reflection with the case of $M=1$, where the charge $q$ induces a dipole in each sphere, and each dipole induces an approximate quadrupole in alternating sphere. The electric fields of a distant dipole and quadrupole drop off with the powers of $1 / r^{2}$ and $1 / r^{4}$; higher order multipoles are with higher order of decay rate and not shown in the figure. From this aspect, the convergence of the series (14) will be fast, which will be verified in next section by numerical experiments.

It is straightforward to extend the approach of constructing images to more dielectric spheres which are separated from each other, where the multiple reflection can be also recursively applied. At first, the source charge will induce images within each sphere due to its dielectric discontinuity. These images then lead to images in other spheres, for which we could decompose the system into pairwise interactions of spheres, and then the above formulas for multipoles can be used to obtain approximate quadrupoles and higher-order multipoles. For example, when three spheres are present, 
the total potential can be written in the same form, $\Phi_{\text {pol }}(\mathbf{r})=\Phi^{(1)}+\Phi^{(2)}+\cdots$, but with the $p$ th order images given by,

$$
\begin{aligned}
\Phi^{(p)}= & \sum_{J=0}^{J_{p-1}}\left(\frac{q_{p, j}^{1}}{\varepsilon_{\mathrm{ex}}\left|\mathbf{r}^{\prime}-\mathbf{x}_{p, j}^{1^{\prime}}\right|}+\right. \\
& \left.\frac{q_{p, j}^{2}}{\varepsilon_{\mathrm{ex}}\left|\mathbf{r}^{\prime \prime}-\mathbf{x}_{p, j}^{2^{\prime \prime}}\right|}+\frac{q_{p, j}^{3}}{\varepsilon_{\mathrm{ex}} \mid \mathbf{r}^{\prime \prime \prime}-\mathbf{x}_{p, j}^{3^{\prime \prime \prime} \mid}}\right)
\end{aligned}
$$

where $J_{p}=2^{p}(M+1)^{p}$, for $p \geq 1$, is the total number of $p$-level images outside one sphere, and $J_{0}=M$. It can be seen the number of images is large, and thus a coarse graining procedure should be proposed for applications of many dielectric spheres, which is not attempted in the present study.

\section{Numerical results}

In this section, the method of images proposed is tested by several numerical examples of two spheres (as we are particularly concerned with the interaction of two colloids with small ions). In all the following calculations, we take $\varepsilon_{i}=2, \varepsilon_{e x}=80$, the temperature $T=298 K$, and the energy unit $\mathrm{kJ} / \mathrm{mol}(1 \mathrm{~kJ} / \mathrm{mol} \approx$ $\left.0.4 k_{B} T\right)$.

To test the accuracy dependence of the different parameters, the number of images $I=M+1$, the number of reflections $P$ and the spacial distance between spheres, we compute the potential energy of a toy model, two separating spheres of radius $R=1 \AA$ immersed in a homogeneous background. A unit point charge is put somewhere outside the spheres. We calculate the self polarization energy of the source charge, which is described by $U=q \Phi_{\mathrm{pol}}\left(\mathbf{r}_{s}\right) / 2$ with $q=1 e$.

For the discretization of a line image, it has been demonstrated only 4 quadrature points can provide very high accuracy. We adopt the results of $I=7$ and $P=8$ as the "exact" reference solution. We not only calculate the errors between the solutions of different parameters with the reference, but also compare them with the solutions numerically computed by solving the 3D Poisson equation. The APBS package (Baker et al., 2001 ) is used to obtain such solutions, which is based on a finite difference (FD) solver together with a multigrid accelerating technique for the numerical algebraic systems. By the FD solver, the Poisson equation is solved twice for the inhomogeneous dielectric media and the homogeneous solvent phase in all space, respectively, and the energy difference of the two cases gives the self polarization energy.

At the first, the two spheres are placed at coordinates $( \pm 1.5,0,0)$, say, the closest distance, $d=1 \AA$. We compute three different sites of the source charge, $\mathbf{r}_{1}=(0,0,0), \mathbf{r}_{2}=(0,0,1)$, and $\mathbf{r}_{3}=(0.4,0,0)$. The first two sites are at the symmetric plane of the two spheres. The last site is very close to one of the spherical surface, which is used to see the performance of the method, as it is difficult for both numerical solvers and the solution based on spherical harmonics. Three groups of parameters are used. Group 1 sets $I=7$ and takes $P$ from 1 and 4 . Group 2 sets $P=8$ but takes varying $I$ from 2 and 5 . Group 3 is the results of the APBS package with different grid sizes $h=0.1 \AA$, $0.05 \AA$ and $0.03 \AA$. The FD solution with $h=0.03 \AA$ is already expensive, where we use $417^{3}$ grids to reduce the influence of inaccurate boundary conditions. The results of energy $U$ of all calculations are listed in Table 1. It is shown the image method is almost convergent at $I=4$ for fixed $P$, and $P=3$ for fixed $I$. For the worst case of $\mathbf{r}_{s}=\mathbf{r}_{3}$, the convergence according to $I$ is slower, but the relative error is already $0.38 \%$ when $I=4$ in Group 2. The image solution is in agreement with the FD solution by APBS. The FD solution is usually considered very accurate in molecular simulations, however, it is evidenced from the table that the convergence of the FD solution is much slower that the image method, in particular, when the source is close to the interface (Site 3).

Table 1 The self polarization energies $(\mathrm{kJ} / \mathrm{mol})$ calculated by the method of images and the APBS package. Groups 1 and 2 are with $I=7$ and $P=8$, respectively. Group 3 is the results of the APBS with different grid sizes $h$

\begin{tabular}{lllll}
\hline & & Site 1 & Site 2 & Site 3 \\
\hline \multirow{4}{*}{ Group 1 } & $P=1$ & 3.5386 & 1.2806 & 25.485 \\
& $P=3$ & 3.7180 & 1.3289 & 25.719 \\
& $P=4$ & 3.7276 & 1.3311 & 25.749 \\
& $I=2$ & 4.7281 & 1.3312 & 25.749 \\
Group 2 & $I=3$ & 3.7357 & 1.4094 & 28.662 \\
& $I=4$ & 3.7284 & 1.3313 & 25.292 \\
& $I=5$ & 3.7282 & 1.3312 & 25.766 \\
Group 3 & $h=0.05$ & 3.8045 & 1.3130 & 20.055 \\
& $h=0.03$ & 3.7877 & 1.3312 & 23.419 \\
"Exact" Reference & 3.7281 & 1.3332 & 28.155 \\
\hline
\end{tabular}

The second concern is the accuracy dependence on the distance between spheres of the same system. We use the closest distance of the two spheres, $d$, as the parameter, and test the performance of the proposed method. Two spherical centers at $( \pm(1+d / 2), 0,0)$ and the source at the origin. We set $I=4$ and three different $P=1,2$ and 3 so that there are totally 8 , 40 and 168 images in two spheres, respectively, and we denote the corresponding energies of the three cases by $U_{8}, U_{40}$ and $U_{168}$. In the fourth case, we use $P=3$ 
and a nonuniform $M$ in each reflection, say, $M=4$ in the first reflection, $M=3$ in the second reflection, and $M=2$ in the third one. The fourth case has totally 80 images, and we denote the results of this case by $U_{80}$. The polarization energies and their relative errors of the four cases are plotted in Fig. 2 for $d$ changing from $0.2 \AA$ to $2.0 \AA$. It can be seen the errors of $U_{168}$ are all less than $1 \%$ even when the two spheres as well as the source charge are very close. $U_{80}$ is also very accurate, which is less than $1 \%$ when $d>0.3 \AA$ (at $d=0.3 \AA$, the absolute error of $U_{80}$ is $0.266 \mathrm{~kJ} / \mathrm{mol}$ ). $U_{80}$ could be a better choice than $U_{168}$ in practical simulations because the number of image is greatly reduced with a small price. When $d$ is a little bit larger, the method with much less images can be considered. For example, the error of $U_{40}$ starts to less than $0.09 \mathrm{~kJ} / \mathrm{mol}$ when $d=0.6 \AA$. When $d>1.2 \AA$, even the method with only 8 images works well, the absolute error of $U_{8}$ is about $0.10 \mathrm{~kJ} / \mathrm{mol}$ for $d=1.2 \AA$ which is a little bit larger than the spherical radius $R$ (at $d=R$, its error is 0.19 $\mathrm{kJ} / \mathrm{mol}$ ). The energies by the APBS with $h=0.05 \AA$ and $321^{3}$ grids are also present for comparison.

\section{Discussion and conclusion}

We have developed a method of images of the surface polarization charge of multiple spheres due to a source in the dielectric background, using the technique of multiple reflections, resulting in a simple, accurate, close-form expression of the polarization potential. The convergence and accuracy dependence on the involved parameters including the number of images, the number of reflections, and the distance between spheres, is investigated, which demonstrates the attractive performance of the method.

The present study shows that the high accuracy treatment of the surface polarization is necessary for simulating particle systems in ionic fluids. Particularly, when the ions are close to the surface of a dielectric, lower order approximations lead to a large deviation of the polarization energy. The benefit of using the method of images is that an any desired accuracy can be achieved by increasing the number of image charges whose parameters are in close form. We found $40 \mathrm{im}$ ages for each source charge can provide very accurate results, though an increase of the number will be better when the distance of two colloids are less than a half of the spherical radius. The polarization due to a charge away from the surface can be represented by a small number of images (for example, 8 images shown in Fig.2, which could be reduced to 4 or 6 images if small ions are away from both spheres), and thus the overall particle number composed the source and image charges are not uncontrollably high for practical simulations.

Furthermore, the particle system of the source and
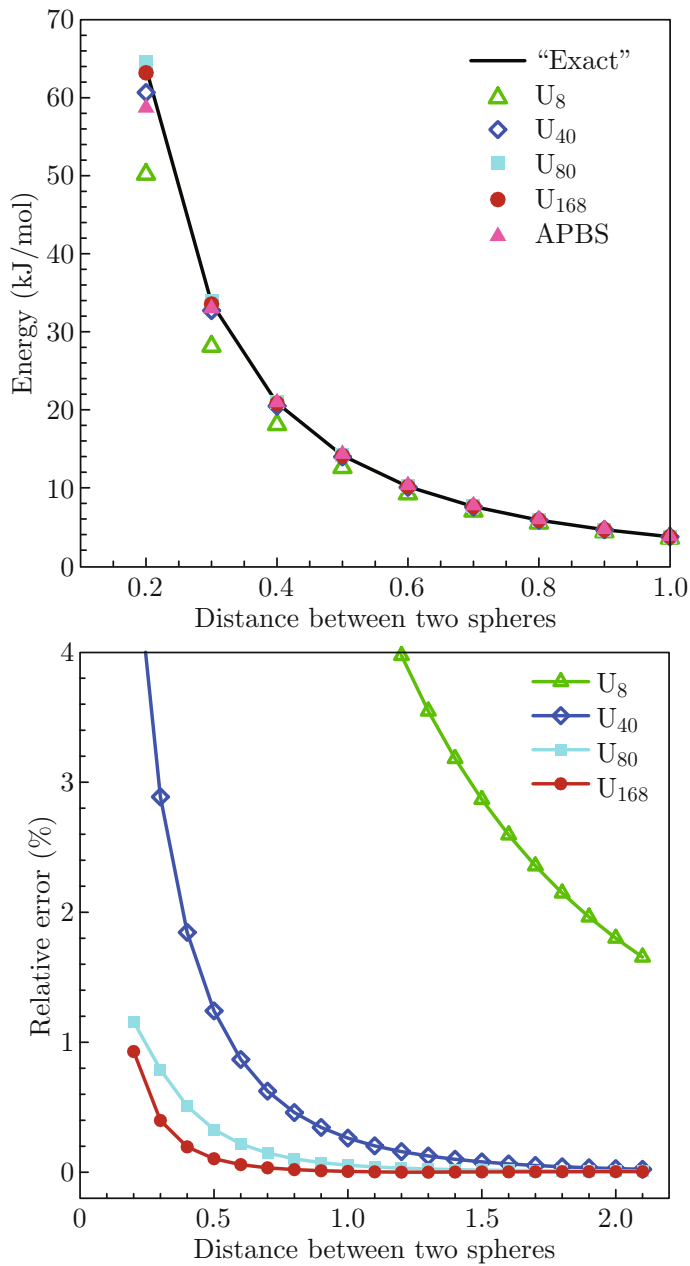

Fig. 2 Self polarization energies of an ion computed by the methods with different numbers of images and their relative errors with the "exact" reference solution, as a function of $d$, the closest distance between two spheres.

image charges is in Coulomb form, which can be treated by the accelerating algorithms such as the FFT-based lattice-sum methods (Arnold and Holm, 2005) which use a periodic boundary condition of the simulation box, and fast multipole methods (Greengard, 1987). The fast multipole algorithms are with an $\mathrm{O}(\mathrm{N})$ scaling, and the breakeven point of the newer version ( $\mathrm{Lu}$ et al., 2006) is dramatically low. The computational performance of the present method could be very much improved once such algorithm is incorporated.

The present method is particularly useful for Monte Carlo and molecular dynamics simulations of soft matter systems where the fast treatment for the dielectric inhomogeneous media is urgent; see a recent application Gan and $\mathrm{Xu}$ (2011) in studying the charge inversion phenomenon of a spherical colloid. The efficient inclusion of surface polarization was limited to the simplest cases such as planar interfaces. Even only planar inter- 
faces are included, the fast lattice-sum methods will systematically slow down. For multiple dielectric spheres, the fast electrostatic calculation has been a challenging problem even when only two spheres are present, which are attracting interest in studying the mechanics of bound pairs of like-charge colloids in aqueous solutions. The method of images we have proposed provides an efficient tool for simulations of these problems which are also the objective of our ongoing project.

Acknowledgements The author acknowledges the financial support from the NSFC (grant numbers: 11101276 and 91130012) and the Chinese Ministry of Education (NCET-09-0556). The author thanks Professor Wei Cai for helpful discussion.

\section{References}

[1] Abagyan, R., Totrov, M. 1994. Biased probability Monte Carlo conformational searches and electrostatic calculations for peptides and proteins. J Mol Biol 235, 983-1002.

[2] Allen, R., Hansen, J.-P. 2002. Density functional approach to the effective interaction between charges within dielectric cavities. J Phys Condens Matter 14, 11981-11997.

[3] Arnold, A., Holm, C. 2005. Efficient methods to compute long-range interactions for soft matter systems. Adv Polym Sci 185, 59-109.

[4] Baker, N.A., Sept, D., Joseph, S., Holst, M.J., McCammon, J.A. 2001. Electrostatics of nanosystems: Application to microtubules and the ribosome. Proc Natl Acad Sci USA 98, 10037-10041.

[5] Bhattacharya, S., Blawzdziewicz, J. 2002. Image system for Stokes-flow singularity between two parallel planar walls. J Math Phys 43, 5720-5731.

[6] Bhuiyan, L.B., Outhwaite, C.W., Henderson, D.J. 2009. Evidence from Monte Carlo simulations for a second contact value theorem for a double layer formed by 2:1/1:2 salts at low electrode charges. Mol Phys 107, 343-347.

[7] Boda, D., Gillespie, D., Nonner, W., Henderson, D., Eisenberg, Bob. 2004. Computing induced charges in inhomogeneous dielectric media: Application in a Monte Carlo simulation of complex ionic systems. Phys Rev E 69, 046702.

[8] Boroudjerdi, H., Kim, Y.-W., Naji, A., Netz, R.R., Schlagberger, X., Serr, A. 2005. Statics and dynamics of strongly charged soft matter. Phys Rep 416, 129199.

[9] Cai, W., Deng, S., Jacobs, D. 2007. Extending the fast multipole method to charges inside or outside a dielectric sphere. J Comput Phys 223, 846-864.

[10] Cheng, H., Greengard, L. 1998. A method of images for the evaluation of electrostatic fields in system of closely spaced conducting cylinders. SIAM J Appl Math 58, 122-141.
[11] Deng, S., Cai, W., Jacobs, D. 2007. A comparable study of image approximations to the reaction field. Comput Phys Commun 177, 689-699.

[12] French, R.H., Parsegian, V.A., Podgornik, R., Rajter, R.F., Jagota, A., Luo, J., Asthagiri, D., Chaudhury, M.K., Chiang, Y., Granick, S., Kalinin, S., Kardar, M. Kjellander, R., Langreth, D.C., Lewis, J., Lustig, S., Wesolowski, D., Wettlaufer, J.S., Ching, W.Y., Finnis, M., Houlihan, F., von Lilienfeld, O.A., van Oss, C.J., Zemb, T. 2010. Long range interactions in nanoscale science. Rev Mod Phys 82, 1887-1944.

[13] Frenkel, D., Smit, B. 2002. Understanding Molecular Simulation: From Algorithms to Applications. Academic Press, New York.

[14] Friedman, H.L. 1975. Image approximation to the reaction field. Mol Phys 29, 1533-1543.

[15] Gan, Z., Xu, Z. 2011. Multiple-image treatment of induced charges in Monte Carlo simulations of electrolytes near a spherical dielectric interface. Phys Rev E 84, 016705.

[16] Greengard, L. 1987. The Rapid Evaluation of Potential Fields in Particle Systems. MIT, Cambridge.

[17] Greengard, L., Rokhlin, V. 1987. A fast algorithm for particle simulations. J Comput Phys 73, 325-348.

[18] Hatlo, M.M., Lue, L. 2008. The role of image charges in the interactions between colloidal particles. Soft Matter 4, 1582-1596.

[19] Jackson, J.D. 2001. Classical Electrodynamics, $3^{\text {rd }}$ Edition. John Wiley \& Sons, New York.

[20] Kirkwood, J.G. 1934. Theory of solutions of molecules containing widely separated charges with special applications to awitterions. J Chem Phys 2, 351-361.

[21] Levin, Y. 2002. Electrostatic corrections: From plasma to biology. Rep Prog Phys 65, 1577-1632.

[22] Lin, Y., Baumketner, A., Deng, S., Xu, Z., Jacobs, D., Cai, W. 2009. An image-based reaction field method for electrostatic interactions in molecular dynamics simulations of aqueous solutions. J Chem Phys 131, 154103.

[23] Lindell, I.V. 1992. Electrostatic image theory for the dielectric sphere. Radio Sci 27, 1-8.

[24] Lindell, I.V. 1993. Application of the image concept in electromagnetism. In: Stone, W.R. (ed), The Review of Radio Science 1990-1992. Oxford University Press, Oxford, Pages 107-126.

[25] Lindell, I.V., Sten, J.C.E., Nikoskinen, K.I. 1993. Electrostatic image theory for the interaction of two dielectric spheres. Radio Sci 28, 319-329.

[26] Linse, P. 1986. Image charge effects in spherical symmetry with applications to micellar systems. J Phys Chem 90, 6821-6828.

[27] Linse, P. 2008. Electrostatics in the presence of spherical dielectric discontinuities. J Chem Phys 128, 214505.

[28] Lotan, I., Head-Gordon, T. 2006. An analytical electrostatic model for salt screened interactions between multiple proteins. J Chem Theory Comput 22, 541555 . 
[29] Lu, B., Cheng, X., Huang, J., McCammon, J.A. 2006. Order $\mathrm{N}$ algorithm for computation of electrostatic interactions in biomolecular systems. Proc Natl Acad Sci USA 103, 19314-19319.

[30] McClurg, R.B., Zukoski, C.F. 1998. The electrostatic interaction of rigid, globular proteins with arbitrary charge distributions. J Colloid Interface Sci 208, 529542 .

[31] Messina, R. 2002. Image charges in spherical geometry: Application to colloidal systems. J Chem Phys 117, 11062.

[32] Messina, R. 2009. Electrostatics in soft matter. J Phys Condens Matter 21, 113101.

[33] Neu, J.C. 1999. Wall-mediated forces between likecharged bodies in an electrolyte. Phys Rev Lett 82, 1072-1074.

[34] Norris, W.T. 1995. Charge images in a dielectric sphere. IEE Proc-Sci Meas Technol 142, 142-150.

[35] Phillies, G.D. 1974. Excess chemical potential of dilute solutions of spherical polyelectrolytes. J Chem Phys 60, 2721-2731.

[36] Qiao, Z., Li, Z., Tang, T. 2006. A finite difference scheme for solving the nonlinear Poisson-Boltzmann equation modeling charged spheres. J Comput Math 24, 252-264.

[37] Rescics, J., Linse, P. 2008. Potential of mean force between charged colloids: Effect of dielectric discontinuities. J Chem Phys 129, 114505.
[38] Torrie, G.M., Valleau, J.P., Patey, G.N. 1982. Electrical double layers. II. Monte Carlo and HNC studies of image effects. J Chem Phys 76, 4615.

[39] Tyagi, S., Suzen, M., Sega, M., Barbosa, M., Kantorovich, S.S., Holm, C. 2010. An iterative, fast, linearscaling method for computing induced charges on arbitrary dielectric boundaries. J Chem Phys 132, 154112.

[40] Wang, Z.Y., Ma, Y.Q. 2009. Monte Carlo determination of mixed electrolytes next to a planar dielectric interface with different surface charge distributions. J Chem Phys 131, 244715.

[41] Xing, X. 2011. The Poisson-Boltzmann theory for the two-plates problem: Some exact results. Interdiscip Sci Comput Life Sci 3, 266-271.

[42] Xu, Z., Cai, W. 2011. Fast analytical methods for macroscopic electrostatic models in biomolecular simulations. SIAM Rev 53, 683-720.

[43] Xu, Z., Cai, W., Cheng, X. 2011. Image charge method for reaction fields in a hybrid ion-channel model. Commun Comput Phys 9, 1056-1070.

[44] Yang, P.K., Liaw, S.H., Lim, C. 2002. Representing an infinite solvent system with a rectangular finite system using image charges. J Phys Chem B 106, 2973-2982.

[45] Yap, E.H., Head-Gordon, T. 2010. New and efficient Poisson-Boltzmann solver for interaction of multiple proteins. J Chem Theory Comput 6, 2214-2224. 